Article

\title{
Median Nerve Neural Mobilization Adds No Additional Benefit When Combined with Cervical Lateral Glide in the Treatment of Neck Pain: A Randomized Clinical Trial
}

\author{
Daniel Martin-Vera ${ }^{1}$, Josué Fernández-Carnero ${ }^{2,3,4,5,6, * \mathbb{D}}$, David Rodríguez-Sanz ${ }^{7}$, Cesar Calvo-Lobo ${ }^{7}$ (D), \\ Ibai López-de-Uralde-Villanueva ${ }^{8}$, Alberto Arribas-Romano ${ }^{2,9}$, Pedro Martínez-Lozano ${ }^{1}$ (D) \\ and Daniel Pecos-Martín 10,11,+ + D
}

1 Faculty of Sport Sciences, Universidad Europea de Madrid, Villaviciosa de Odón, 28005 Madrid, Spain; daniel.martinvera@gmail.com (D.M.-V.); pedro.martinez@universidadeuropea.es (P.M.-L.)

2 Department of Physical Therapy, Occupational Therapy, Rehabilitation and Physical Medicine, Rey Juan Carlos University, 28922 Madrid, Spain; alberto.arribas@urjc.es

3 La Paz Hospital Institute for Health Research, IdiPAZ, 261, 28046 Madrid, Spain

4 Grupo Multidisciplinar de Investigación y Tratamiento del Dolor, Grupo de Excelencia Investigadora, URJC-Banco de Santander, 28922 Madrid, Spain

5 Motion in Brains Research Group, Institute of Neuroscience and Movement Sciences (INCIMOV), Centro Superior de Estudios Universitarios La Salle, Universidad Autonóma de Madrid, 28049 Madrid, Spain

check for updates

Citation: Martin-Vera, D.; Fernández-Carnero, J.; RodríguezSanz, D.; Calvo-Lobo, C.; López-deUralde-Villanueva, I.; ArribasRomano, A.; Martínez-Lozano, P.; Pecos-Martín, D. Median Nerve Neural Mobilization Adds No Additional Benefit When Combined with Cervical Lateral Glide in the Treatment of Neck Pain: A Randomized Clinical Trial. J. Clin Med. 2021, 10, 5178. https:// doi.org/10.3390/jcm10215178

Academic Editor: Tomasz Halski

Received: 18 October 2021

Accepted: 1 November 2021

Published: 5 November 2021

Publisher's Note: MDPI stays neutral with regard to jurisdictional claims in published maps and institutional affiliations.

Copyright: (c) 2021 by the authors. Licensee MDPI, Basel, Switzerland. This article is an open access article distributed under the terms and conditions of the Creative Commons Attribution (CC BY) license (https:// creativecommons.org/licenses/by/ $4.0 /)$.
6 Grupo de Investigación de Dolor musculoesqueletico y Control Motor, Universidad Europea de Madrid, 28005 Madrid, Spain

7 School of Nursing, Physiotherapy and Podiatry, Universidad Complutense de Madrid, 28606 Madrid, Spain; davidrodriguezsanz@ucm.es (D.R.-S.); cescalvo@ucm.es (C.C.-L.)

8 Department of Radiology, Rehabilitation and Physiotherapy, Universidad Complutense de Madrid, 28606 Madrid, Spain; ibailope@ucm.es

9 Escuela Internacional de Doctorado, Department of Physical Therapy, Occupational Therapy, Rehabilitation and Physical Medicine, Universidad Rey Juan Carlos, 28933 Alcorcón, Spain

10 Physiotherapy and Pain Research Center, General Foundation of the University of Alcalá, 28805 Madrid, Spain; daniel.pecos@uah.es

11 Department of Physical Therapy, Alcalá University, 28805 Alcalá de Henares, Spain

* Correspondence: josue.fernandez@urjc.es; Tel.: +34-914888949

+ Membership of the Physiotherapy and Pain Group.

Abstract: Background: This study aimed to compare the effectiveness of cervical lateral glide (CLG) added to median nerve neural mobilization (MNNM) in patients with neck pain (NP). Methods: A single-blinded randomized controlled clinical trial was carried out in a Pain Management Unit from a Hospital. A total sample of 72 patients with NP was recruited from a hospital. Patients were randomized to receive isolated CLG $(n=36)$ or CLG $+\operatorname{MNNM}(n=36)$. Bilateral elbow extension range of motion (ROM) on upper limb neurodynamic test 1 (ULNT1), bilateral pressure pain thresholds (PPT) on the median nerve at elbow joint, $\mathrm{C}_{6}$ zygapophyseal joint and tibialis anterior, Visual analogue scale (VAS), body chart distribution of pain, active cervical ROM (CROM), Neck Disability Index (NDI), and Tampa Scale of Kinesiophobia (TSK-11) were measured at baseline as well as immediately, 15 days, and 1 month after treatment. Results: There were no statistically significant interactions $(p>0.05)$ between treatment and time for median nerve mechanosensitivity outcomes, pain intensity, symptom distribution, and PPT of the widespread pain assessment, as well as cervical function, and kinesiophobia. Conclusions: MNNM gave no additional benefit to CLG in patients with NP regarding pain intensity, symptom distribution, mechanosensitivity, functionality, and kinesiophobia. Only two treatment sessions and the short follow-up are important issues, therefore, justifying further studies to answer the research question with better methodology.

Keywords: brachial plexus neuritis; chronic neck pain; musculoskeletal manipulations; rehabilitation; upper extremity 


\section{Introduction}

Neck pain (NP) is a common disabling condition that is likely to affect most people at some time in their lives [1-3]. NP might negatively impact the social, familial, and work environments of the patients, as well as affect the health care system and contribute to economic burden [1,4-6]. The NP global point prevalence was $4.9 \%$ and the disabilityadjusted life years showed an increase from 23.9 million in 1990 to 33.6 million in 2010. NP is ranked fourth among years lived with disability and 21st when considering the overall burden [3]. Indeed, the prevalence of NP has risen to $8.56 \%$ in Spain and reached $10.61 \%$ in conjunction with low back pain [1].

Neuropathic features might be present in $6.9 \%$ to $10 \%$ of patients with NP [7]. Indeed, neuropathic pain frequency might increase by up to $19.9 \%$ in patients with NP associated with pain in the arm (cervicobrachial pain (CBP)) [8]. Pain management among patients with nociceptive or neuropathic NP can be complex. Thus, the appropriate interventions to treat these patients depend on the nature of pain [9]. Although different interventions have been proposed for nociceptive $[10,11]$ and neuropathic [12-14] NP, there is low evidence to determine the effectiveness of physical medicine modalities, such as neural mobilization, and high quality randomized clinical trials are necessary for patients with NP $[15,16]$.

Regarding neural mobilization approaches for musculoskeletal conditions in patients with CBP $[15,16]$, median nerve neural mobilization (MNNM) and cervical lateral glide (CLG) were shown to be more effective in short-term for improving pain intensity, range of motion (ROM) during upper limb neurodynamic test (ULNT1), and functionality than therapeutic ultrasound [17], manual cervical traction [18] or no treatment [19,20]. Although pharmacologic treatment, such as oral ibuprofen, can decrease pain intensity and disability more than MNNM or CLG, the non-existence of ROM differences between these interventions, as well as adverse effects associated with the pharmacological agents, suggest neural mobilization as a possible treatment option in patients with CBP [21,22]. Indeed, neural mobilization techniques for upper quadrant pain syndromes might modulate central sensitization and mechanosensitivity in patients with NP [23].

To our knowledge, the effectiveness of combining MNNM with CLG has not yet been determined in patients with NP. This study aimed to compare the effectiveness of CLG plus MNNM (vs. MNNM alone) on median nerve mechanosensitivity, intensity and distribution of NP, local/remote pressure pain threshold (PPT), cervical function, and kinesiophobia in patients with NP.

\section{Materials and Methods}

\subsection{Trial Design}

The study protocol was conducted from April to June of 2017 as an interventional phase II, parallel, single-blind, randomized controlled clinical trial. The study was conducted at the Pain Management Unit from the Alcala de Henares Hospital (Spain) in accordance with the CONSORT statement [24].

All of the procedures used in this trial were programmed according to the ethical principles of the Declaration of Helsinki [25]. All subjects were informed and provided informed consent to participate. This trial was approved by the Ethics Committee for Clinical Research of the Principe de Asturias Hospital (Code: OE 19/2013). This trial was prospectively registered in the Australian New Zealand Clinical Trials Registry (ACTRN12c617000430336).

\subsection{Randomization and Blinding}

The randomization and allocation of the patients to the trial group were done using computer software (Epidat ${ }^{\circledR} 4.2$ version, Xunta de Galicia, Spain) and randomized printed cards contained in consecutively numbered opaque sealed envelopes kept in a locked drawer unit. A member of the research team who was not involved in the assessment and/or treatment procedures performed the randomization and maintenance of the list. 
Treatment and outcome assessments were performed in different rooms and at different time points.

\subsection{Follow-Up}

The outcomes assessment was carried out at baseline, post-intervention, and 15 days and 30 days after the first intervention. Median nerve mechanosensitivity, pain-related outcomes, and cervical ROM were recorded in all evaluation time spots. Neck Disability Index (NDI) and the Tampa Scale of Kinesiophobia (TSK-11) measurements were not obtained at post-intervention. Sociodemographic data were also collected.

\subsection{Median Nerve Mechanosensitivity}

ROM in elbow extension on the ULNT1 measures was performed [26]. In the ULNT1 maneuver for fixing the shoulder girdle, wrist, and hand [26-29], patients needed to specify the moment in which sub-maximum pain level was reached with elbow extension, which means the level of pain that subjects can tolerate. Elbow extension was assessed using a universal goniometer (Sammons Preston ${ }^{\circledR}$, Bolingbrook, IL, USA). UNLT1 Intra and inter-observer reliability is considered from good to excellent (ICC $\geq 0.98$ ) [30]. An average of three measurements was calculated.

\subsection{Widespread Pain Assessment}

2.5.1. Pain Intensity

Pain intensity levels were recorded with a VAS, described as a $100 \mathrm{~mm}$ non-segmented line, oriented horizontally, with both extremes labeled as "no pain" at its start and finishing with "the worst pain unimaginable". Subjects were asked to rate their current pain with a mark on the scale [31]. VAS is a reliable (ICC) intraclass correlation coefficient and valid tool to measure pain levels. Test-retest reliability is high (ICC 0.71-0.99) [32,33].

\subsubsection{Body Pain Distribution}

Pain/Symptoms distribution data was gathered using four body charts depicting the neck and upper limbs and all drawings were converted to a digital format. The percentage of symptoms change over time was analyzed using Matlab ${ }^{\circledR}$ R2016a version (The MathWorks Inc, Natick, MA, USA) [34]. ICC for distribution of symptoms over the neck region is very high (ICC $=0.92)$, and the software error measured between two exact figures was $5.4 \%$, with good test-retest reliability [34].

\subsubsection{Pressure Pain threshold (PPT)}

PPT was used to quantitatively evaluate local and central tissue mechanosensitivity [33,35-38]. The PPT is defined as the lowest pressure that needs to be applied to cause the slightest sensation of pain [39]. Recordings were collected bilaterally over the articular pillar of the C6 zygapophyseal joint, median nerve at the cubital fossa of the elbow, and tibialis anterior muscle belly $(5 \mathrm{~cm}$ distal from anterior tibial tuberosity and $2.5 \mathrm{~cm}$ lateral) [40-43]. A pressure algometer [FORCE DIAL FDK/FDN 100 model, Wagner Instruments (P.O.B. 1217, Greenwich, CT, USA)] was used $\left(\mathrm{kg} / \mathrm{cm}^{2}\right)$. ICC for the test-retest reliability of PPT in patients with NP are high (ICC: 0.83 to 0.89) [44,45]. In addition, the intra-examiner reliability (ICC $=0.94-0.97$ ) is excellent [46]. An average of three measurements was calculated [47].

\subsection{Cervical Function}

Active cervical ROM was assessed with a CROM device (Cervical Range of Motion Instrument, Performance Attainment Associates, Roseville, MN, USA) through a standardized protocol. Patients were seated on a chair with the CROM device placed over the head. The assessor asked patients to perform active neck movements in the maximum range. ICC values are high for both intra-observer (ICC 0.92-0.96) and inter-observer (ICC 0.82-0.94) 
reliability. Among NP patients, SE has been reported as between $2.5^{\circ}$ and $4.1^{\circ}$ [48]. Three measurements were performed in each direction, and the average values were calculated.

Neck disability was measured with the NDI, which is a well-validated 10-item questionnaire, with each item rated on a 0 to 5 point scale. The highest results correspond to elevated levels of disability [49], NDI is the most used and validated instrument to measure disability levels in non-specific NP patients with or without irradiation symptoms [50,51]. A Spanish version of the NDI was utilized in this study [52].

\subsection{TSK-11}

TSK-11 was selected in this trial to quantify fear of movement behavior or vulnerability related to NP [53]. A validated and reliable Spanish version of the TSK-11 was used [54]. This version has demonstrated good psychometric properties [55].

\subsection{Participants}

Patients with NP were included in the study if they met the following inclusion criteria: between 18-65 years old; NP from non-traumatic origin with a clinical evolution of a minimum of 4 weeks [56] located from the superior nuchal line to the drawn segment between the superomedial angles of the scapula, with or without radicular symptoms radiated to the head, trunk, and/or the upper limbs [57]; and needed to understand, write, and speak Spanish fluently. Exclusion criteria following a clinical evaluation were: two or more abnormal neurological findings within C5-T1 levels; and central or peripheral neurological syndromes; pregnancy and patients that have received physiotherapy at the spine or glenohumeral region over the last 6 weeks.

\subsection{Intervention}

Each subject came to the center for 3 days. Four evaluation points and two interventions were carried out. These interventions were performed at the beginning of the trial and 2 weeks after.

Subjects were randomly and equitably allocated into two groups. A physiotherapist with more than 8 years of experience in manual therapy and neurodynamic techniques. Both groups received treatment of four series of Maitland grade 3 mobilizations [58] in $1 \mathrm{~min}$ with $1 \mathrm{~min}$ of rest between sets at a frequency of $0.5 \mathrm{~Hz}$ (with metronome control/steps). Both groups were taught to do the same neurodynamic exercises of median nerve sliding with movements of the head and elbow simultaneously as a home treatment program between sessions. Assurance of performance of home exercises was supported by an explanatory video sent by email and reminder calls from the trial staff to clarify doubts and motivate about the importance of completing the program [59]. The home exercises pattern was three sets of $1 \mathrm{~min}$ with $1 \mathrm{~min}$ of rest every day.

\subsection{Cervical Lateral Glide and Median Nerve Mobilization (CLG + MNNM)}

This group received a treatment of cervical lateral glide contralateral to the side where each subject suffered higher pain intensity. Patients lay in a supine position on a stretcher and ULNT1 was performed on the upper limb until subjects felt tension on the anterior part of the forearm or hand. Shoulder depression was controlled by a wooden device covered with a soft cushioning, preventing the elevation of the glenohumeral joint, as well as a wrist and thermoplastic splint held hand extension. The physiotherapist performed a contralateral cervical glide with both hands while the patient synchronously slightly flexed the elbow joint. When the therapist returned to the midline, the patients extended the elbow back to the starting position. 


\subsection{Cervical Lateral Glide in Isolation (Isolated CLG)}

Like the previous group, this group received an intervention of cervical lateral glide but with either upper limb in the resting position, placing on the abdominal region.

\subsection{Sample Size}

The sample size was calculated using G*Power 3.0.18 Software (Bonn, Germany). A repeated-measures analysis of variance (ANOVA) was used to detect differences in the median nerve mechanosensitivity. A significance level of $5 \% \alpha$ error and a power of $90 \%$ $(1-\beta$ error) were also used. Moreover, a moderate effect size $(f=0.2)$ and sphericity correction of 0.75 were considered based on a previous pilot study. Assuming a $20 \%$ dropout rate, it was estimated that 68 participants were required (34 per group).

\subsection{Statistical Analysis}

Descriptive statistics were used to describe the baseline characteristics of each group. No statistically significant differences between groups were found. Were examined for normality using the Kolmogorov-Smirnov test for all variables, confirming that all variables were normally distributed. However, two-way ANCOVA was performed to control for the age of the participants because this variable showed differences between groups at baseline to median nerve mechanosensitivity, PPT, ROM, and self-administration questionnaires. The between-subject factor was the treatment (CLG + MNM treatment, CLG treatment), with time (baseline, 2 min immediately post-treatment, 2 weeks follow-up, 4 weeks followup) as within-subject factors. The effect size was calculated as the Partial Eta Squared $\left(\eta^{2} p\right)$ when significant. An effect size of 0.01 was considered small, 0.06 medium, and 0.14 large. A multiple comparisons analysis with Bonferroni adjustment was performed. Statistical analyses were performed using SPSS 22 (SPSS Inc, Chicago, IL, USA). The significance level was set at $p<0.05$.

\section{Results}

The patients and the final inclusion are represented in the flow diagram (Figure 1). We initially recruited 165 patients, but finally, a total of 72 patients were definitively analyzed (29 males/43 females). Finally, 36 subjects completed the study in the cervical lateral glide (CLG) group and 36 CLG + median nerve mobilization (CLG + MNM) group. All of these participants completed the two treatment sessions, including the home task performance of neural mobilizations. The baseline characteristics of both groups are presented in Table 1 . There were no significant differences in baseline variables between the groups $(p>0.05)$, except age (Table 1).

\subsection{Median Nerve Mechanosensitivity}

By two-way ANCOVA, we detected statistically significant differences only in the group factor for the contralateral median nerve PPTs $\left(F=5.767 ; p=0.019 ; \eta^{2} p=0.078\right)$. There were no statistically significant interactions between treatment and time for median nerve mechanosensitivity outcomes [homolateral ULNT1 $\left(\mathrm{F}=1.118 ; p=0.332 ; \eta^{2} \mathrm{p}=0.016\right)$; contralateral ULNT1 $\left(\mathrm{F}=0.346 ; p=0.747 ; \eta^{2} \mathrm{p}=0.005\right)$; homolateral median nerve PPTs $\left(\mathrm{F}=0.741 ; p=0.511 ; \eta^{2} \mathrm{p}=0.011\right)$; contralateral median nerve PPTs $(\mathrm{F}=0.140 ; p=0.890$; $\left.\left.\eta_{p}^{2}=0.002\right)\right]$. All outcomes for median nerve mechanosensitivity are presented in Table 2 . 

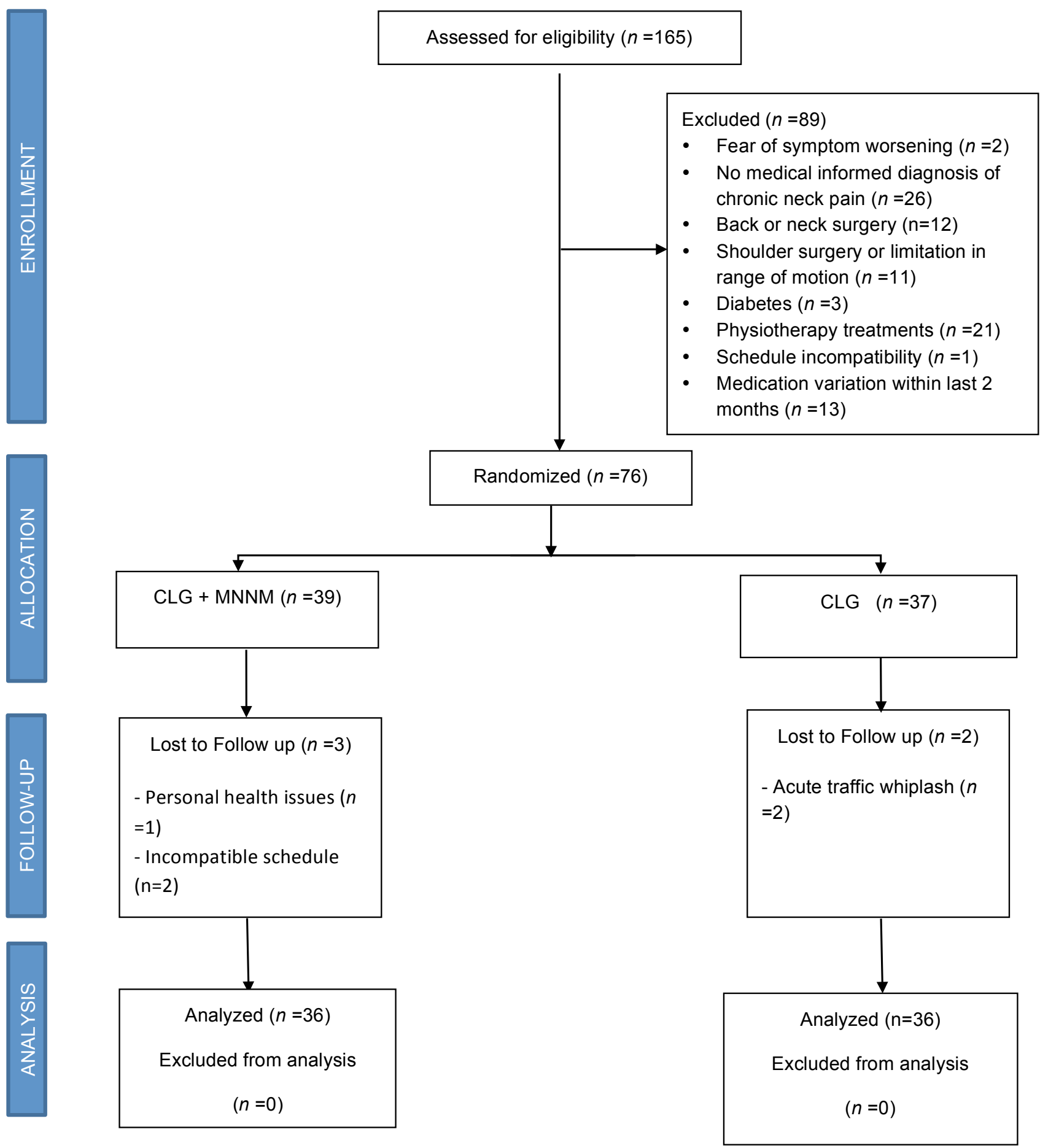

Figure 1. Consort flow diagram. CLG = cervical Lateral Glide; $M N N M=$ median nerve neural mobilization. 


\subsection{Widespread Pain Assessment}

There were significant differences for time factor in the PPT for the homolateral C6 zygapophyseal joint PPT $\left(\mathrm{F}=4.707 ; p=0.006 ; \eta^{2} \mathrm{p}=0.065\right)$. However, no differences were found in the group $\times$ time interaction for pain intensity, body pain distribution, and PPT. Post hoc analysis for pain intensity, body pain distribution, and PPT are shown in Table 3.

Table 1. Baseline participant characteristics. Values are the mean \pm standard deviation.

\begin{tabular}{|c|c|c|c|}
\hline & $\begin{array}{c}\text { CLGS + MNNM Group } \\
n=(72)\end{array}$ & $\begin{array}{c}\text { CLGS Group } \\
\quad n=(72)\end{array}$ & $p$-Value \\
\hline Age, years & $44.69 \pm 13.48$ & $50.72 \pm 9.42$ & $0.03 *$ \\
\hline Sex M/F (Female\%) & $15 / 21(48.8 \%)$ & $14 / 22(51.2 \%)$ & 0.81 \\
\hline Duration of pain (months) & $73.11 \pm 53.52$ & $61.61 \pm 42.09$ & 0.31 \\
\hline PPT-C6-H $\left(\mathrm{kg} / \mathrm{cm}^{2}\right)$ & $2.48 \pm 1.14$ & $2.58 \pm 1.09$ & 0.71 \\
\hline PPT-C6-CL (kg/cm²) & $2.53 \pm 0.83$ & $2.74 \pm 0.98$ & 0.34 \\
\hline PPT-MN-H (kg/cm²) & $2.82 \pm 0.95$ & $2.91 \pm 1.16$ & 0.74 \\
\hline PPT-MN-CL (kg/ $\left.\mathrm{cm}^{2}\right)$ & $2.71 \pm 1.01$ & $3.03 \pm 1.08$ & 0.20 \\
\hline PPT-AT-H $\left(\mathrm{kg} / \mathrm{cm}^{2}\right)$ & $7.41 \pm 3.40$ & $7.19 \pm 3.17$ & 0.77 \\
\hline PPT-AT-CL $\left(\mathrm{kg} / \mathrm{cm}^{2}\right)$ & $6.99 \pm 3.34$ & $7.01 \pm 3.20$ & 0.97 \\
\hline Neck-PD & $8.45 \pm 6.79$ & $8.98 \pm 11.67$ & 0.81 \\
\hline UL-PD-HL & $5.91 \pm 8.38$ & $6.24 \pm 12.58$ & 0.89 \\
\hline UL-PD-CL & $2.24 \pm 4.43$ & $2.63 \pm 8.07$ & 0.79 \\
\hline Elbow extension & $49.50 \pm 19.9$ & $44.36 \pm 21.07$ & 0.29 \\
\hline NDI (0 to 50) & $33.69 \pm 16.64$ & $34.83 \pm 16.48$ & 0.77 \\
\hline $\begin{array}{l}\text { VAS (0 to } 100 \mathrm{~mm}) \\
\text { CROM (grades) }\end{array}$ & $42.86 \pm 19.13$ & $42.78 \pm 21.33$ & 0.98 \\
\hline Flexion/extension & $105.77 \pm 29.59$ & $102.30 \pm 24.55$ & 0.59 \\
\hline Lateral flexion & $69.53 \pm 22.25$ & $66.97 \pm 17.35$ & 0.58 \\
\hline Rotation & $115.58 \pm 30.85$ & $115.52 \pm 20.99$ & 0.99 \\
\hline \multicolumn{4}{|l|}{ Psychological measures } \\
\hline TSK-11 (11 to 44$)$ & $27.31 \pm 7.73$ & $28.92 \pm 7.94$ & 0.38 \\
\hline
\end{tabular}

Abbreviations: CLGS + MNNM group, cervical lateral glide-side + median nerve neural mobilization; CLGS group, cervical lateral glide-side; PPT-C6-HL, pressure pain threshold on C6 homolateral side; PPT-C6-CL, pressure pain threshold on $\mathrm{C} 6$ contralateral side; PPT-AT-H, pressure pain threshold on anterior tibial muscle homolateral side; PPT-AT-CL, pressure pain threshold on anterior tibial muscle contralateral side; VAS, visual analogue scale; SD, standard deviation NDI, neck disability index; CROM, cervical range of motion; 11, tampa scale of kinesiophobia; Neck-PD, pain drawing on neck; UL-PD_HL, upper limb pain drawing homolateral; UL-PD-CL, upper limb pain drawing contralateral. $p<0.01 .{ }^{*}=p$ value $<0.05$.

\subsection{Cervical Function and Kinesiophobia}

The ANCOVA analysis only found statistically significant differences in the time factor for kinesiophobia $\left(F=8.013 ; p=0.001 ; \eta^{2} p=0.105\right)$. However, no statistically significant differences were observed in the group $\times$ time interaction for the cervical function and kinesiophobia. Multiple comparisons for the active cervical ROM, disability, and kinesiophobia are presented in Tables 4 and 5. 
Table 2. Multiple comparisons for median nerve mechanosensitivity. Values are the mean \pm standard deviation unless otherwise indicated.

\begin{tabular}{|c|c|c|c|c|c|c|c|c|c|}
\hline & & & & & & Mea & In Difference (95\% CI) & $\mathrm{Me}$ & In Difference (95\% CI) \\
\hline & Group & Baseline & $\begin{array}{l}\text { Post-Intervention } \\
5 \mathrm{~min}\end{array}$ & $\begin{array}{l}\text { Follow-up } \\
2 \text { Weeks }\end{array}$ & $\begin{array}{l}\text { Follow-up } \\
4 \text { Weeks }\end{array}$ & $\begin{array}{l}\text { (a) } \\
\text { (b) } \\
\text { (c) }\end{array}$ & $\begin{array}{l}\text { Baseline vs Post } \\
\text { Baseline vs 2-Weeks } \\
\text { Baseline vs 4-Weeks }\end{array}$ & $\begin{array}{l}\text { (a) } \\
\text { (b) } \\
\text { (c) }\end{array}$ & $\begin{array}{l}\text { Post vs 2-Weeks } \\
\text { Post vs } 4 \text {-Weeks } \\
\text { 2-Weeks vs } 4 \text {-Weeks }\end{array}$ \\
\hline \multicolumn{10}{|c|}{ ULNT1 (degrees for elbow extension) } \\
\hline $\begin{array}{l}\text { Homolateral } \\
\text { side pain }\end{array}$ & CLG + MNNM & $50.85 \pm 20.78$ & $47.09 \pm 20.02$ & $40.80 \pm 19.72$ & $37.31 \pm 18.47$ & $\begin{array}{l}\text { (a) } \\
\text { (b) } \\
\text { (c) }\end{array}$ & $\begin{array}{l}3.76(-0.77 \text { to } 8.29) \\
10.05(1.54 \text { to } 18.56) * \\
13.55(5.74 \text { to } 21.35) * *\end{array}$ & $\begin{array}{l}\text { (a) } \\
\text { (b) } \\
\text { (c) }\end{array}$ & $\begin{array}{l}6.29(-1.53 \text { to } 14.11) \\
9.78(3.10 \text { to } 16.47) * * \\
3.49(-2.47 \text { to } 9.46)\end{array}$ \\
\hline \multirow{2}{*}{$\begin{array}{l}\text { Contralateral } \\
\text { side pain }\end{array}$} & CLG + MNNM & $45.96 \pm 18.80$ & $47.13 \pm 18.28$ & $38.53 \pm 15.98$ & $34.57 \pm 16.63$ & $\begin{array}{l}\text { (a) } \\
\text { (b) } \\
\text { (c) }\end{array}$ & $\begin{array}{l}-1.17(-6.92 \text { to } 4.58) \\
7.43(-1.30 \text { to } 16.16) \\
11.38(3.62 \text { to } 19.15)\end{array}$ & $\begin{array}{l}\text { (a) } \\
\text { (b) } \\
\text { (c) }\end{array}$ & $\begin{array}{l}8.60(0.64 \text { to } 16.56) * \\
12.56(5.15 \text { to } 19.97) * * \\
3.96(-2.45 \text { to } 10.36)\end{array}$ \\
\hline & CLG & $43.24 \pm 19.27$ & $39.20 \pm 18.73$ & $38.27 \pm 16.38$ & $32.88 \pm 17.05$ & $\begin{array}{l}\text { (a) } \\
\text { (b) } \\
\text { (c) }\end{array}$ & $\begin{array}{l}4.04(-1.85 \text { to } 9.94) \\
4.97(-3.98 \text { to } 13.92) \\
10.37(2.41 \text { to } 18.33)^{* *}\end{array}$ & $\begin{array}{l}\text { (a) } \\
\text { (b) } \\
\text { (c) }\end{array}$ & $\begin{array}{l}0.93(-7.23 \text { to } 9.10) \\
6.32(-1.27 \text { to } 13.92) \\
5.39(-1.17 \text { to } 11.96) \\
\end{array}$ \\
\hline \multirow{2}{*}{$\begin{array}{l}\text { Homolateral } \\
\text { side pain }\end{array}$} & CLG + MNNM & $2.76 \pm 1.06$ & $2.82 \pm 1.15$ & $2.89 \pm 1.23$ & $3.20 \pm 1.36$ & $\begin{array}{l}\text { (a) } \\
\text { (b) } \\
\text { (c) }\end{array}$ & $\begin{array}{l}-0.06(-0.39 \text { to } 0.27) \\
-0.13(-0.61 \text { to } 0.35) \\
-0.44(-0.95 \text { to } 0.07)\end{array}$ & $\begin{array}{l}\text { (a) } \\
\text { (b) } \\
\text { (c) }\end{array}$ & $\begin{array}{l}-0.07(-0.53 \text { to } 0.39) \\
-0.38(-0.85 \text { to } 0.09) \\
-0.31(-0.77 \text { to } 0.15)\end{array}$ \\
\hline & CLG & $2.81 \pm 1.09$ & $2.98 \pm 1.18$ & $3.19 \pm 1.26$ & $3.77 \pm 1.39$ & $\begin{array}{l}\text { (a) } \\
\text { (b) } \\
\text { (c) }\end{array}$ & $\begin{array}{l}-0.17(-0.51 \text { to } 0.17) \\
-0.38(-0.87 \text { to } 0.12) \\
-0.96(-1.48 \text { to }-0.43)^{* *}\end{array}$ & $\begin{array}{l}\text { (a) } \\
\text { (b) } \\
\text { (c) }\end{array}$ & $\begin{array}{l}-0.21(-0.68 \text { to } 0.27) \\
-0.79(-1.27 \text { to }-0.31)^{* *} \\
-0.58(-1.05 \text { to }-0.11)^{* *}\end{array}$ \\
\hline \multirow{2}{*}{$\begin{array}{l}\text { Contralateral } \\
\text { side pain }\end{array}$} & CLG + MNNM & $2.67 \pm 1.03$ & $2.81 \pm 1.04$ & $3.04 \pm 1.08$ & $3.53 \pm 1.43$ & $\begin{array}{l}\text { (a) } \\
\text { (b) } \\
\text { (c) }\end{array}$ & $\begin{array}{l}-0.15(-0.47 \text { to } 0.18) \\
-0.37(-0.84 \text { to } 0.09) \\
-0.86(-1.45 \text { to }-0.28)\end{array}$ & $\begin{array}{l}\text { (a) } \\
\text { (b) } \\
\text { (c) }\end{array}$ & $\begin{array}{l}-0.23(-0.70 \text { to } 0.24) \\
-0.72(-1.31 \text { to }-0.12) \text { * } \\
-0.49(-0.96 \text { to }-0.01) \text { * }\end{array}$ \\
\hline & CLG & $2.90 \pm 1.06$ & $3.13 \pm 1.07$ & $3.33 \pm 1.11$ & $3.76 \pm 1.47$ & $\begin{array}{l}\text { (a) } \\
\text { (b) } \\
\text { (c) }\end{array}$ & $\begin{array}{l}-0.23(-0.56 \text { to } 0.10) \\
-0.44(-0.91 \text { to } 0.04) \\
-0.86(-1.47 \text { to }-0.26)\end{array}$ & $\begin{array}{l}\text { (a) } \\
\text { (b) } \\
\text { (c) }\end{array}$ & $\begin{array}{l}-0.21(-0.69 \text { to } 0.28) \\
-0.64(-1.24 \text { to }-0.03) * \\
-0.43(-0.92 \text { to } 0.06)\end{array}$ \\
\hline
\end{tabular}

Abbreviations: CLG, cervical lateral glide; MNNM, median nerve neural mobilization; PPT, pressure pain threshold; ULNT1, upper limb neurodynamic test. ${ }^{*}=p$ value $<0.05,{ }^{* *}=p$ value $<0.01$. 
Table 3. Multiple comparisons for pain intensity, symptom distribution and pressure pain thresholds. Values are the mean \pm standard deviation unless otherwise indicated.

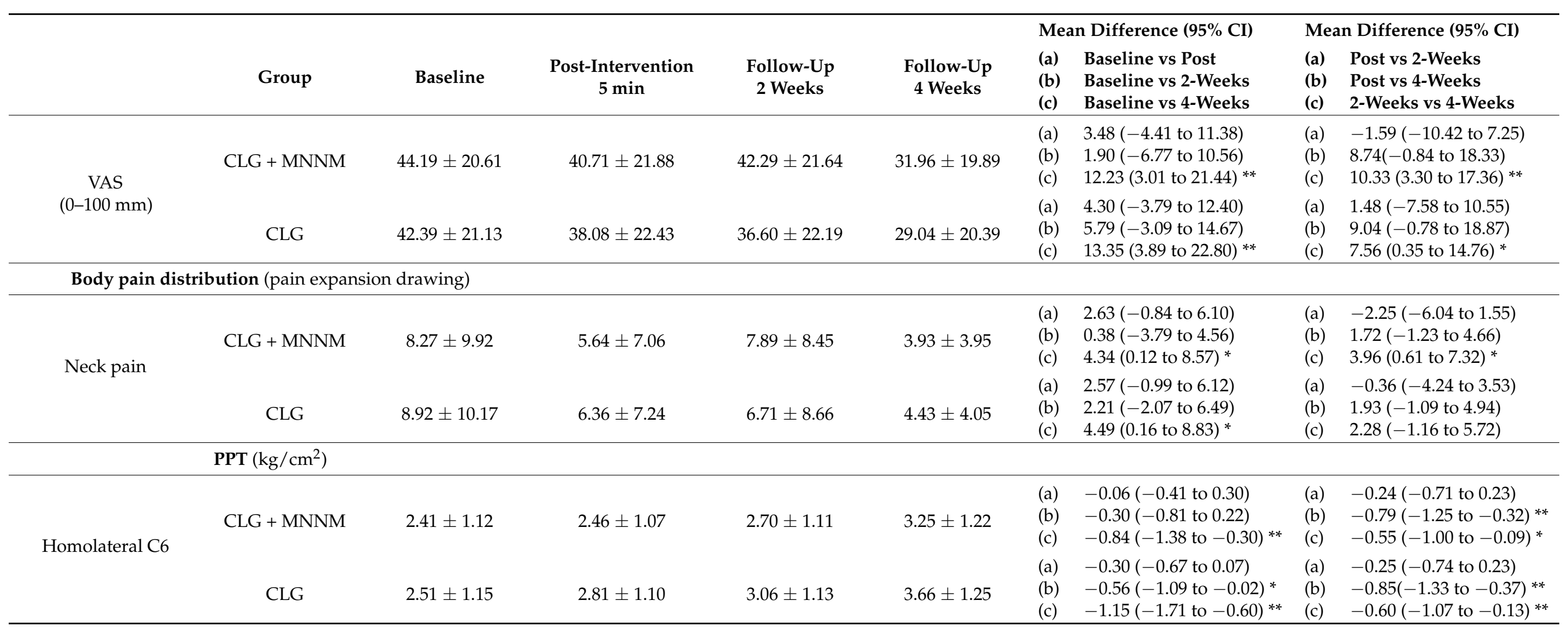


Table 3. Cont.

\begin{tabular}{|c|c|c|c|c|c|c|c|c|c|}
\hline & \multirow[b]{2}{*}{ Group } & \multirow[b]{2}{*}{ Baseline } & \multirow[b]{2}{*}{$\begin{array}{l}\text { Post-Intervention } \\
5 \mathrm{~min}\end{array}$} & \multirow[b]{2}{*}{$\begin{array}{l}\text { Follow-Up } \\
2 \text { Weeks }\end{array}$} & \multirow[b]{2}{*}{$\begin{array}{l}\text { Follow-Up } \\
4 \text { Weeks }\end{array}$} & \multicolumn{2}{|c|}{ Mean Difference (95\% CI) } & \multicolumn{2}{|c|}{ Mean Difference (95\% CI) } \\
\hline & & & & & & $\begin{array}{l}\text { (a) } \\
\text { (b) } \\
\text { (c) }\end{array}$ & $\begin{array}{l}\text { Baseline vs Post } \\
\text { Baseline vs 2-Weeks } \\
\text { Baseline vs } 4 \text {-Weeks }\end{array}$ & $\begin{array}{l}\text { (a) } \\
\text { (b) } \\
\text { (c) }\end{array}$ & $\begin{array}{l}\text { Post vs 2-Weeks } \\
\text { Post vs } 4 \text {-Weeks } \\
\text { 2-Weeks vs } 4 \text {-Weeks }\end{array}$ \\
\hline \multirow{2}{*}{ Contralateral C6 } & CLG + MNNM & $2.47 \pm 0.91$ & $2.63 \pm 1.15$ & $2.80 \pm 0.97$ & $3.40 \pm 1.27$ & $\begin{array}{l}\text { (a) } \\
\text { (b) } \\
\text { (c) }\end{array}$ & $\begin{array}{l}-0.15(-0.48 \text { to } 0.18) \\
-0.33(-0.76 \text { to } 0.10) \\
-0.93(-1.44 \text { to }-0.42)\end{array}$ & $\begin{array}{l}\text { (a) } \\
\text { (b) } \\
\text { (c) }\end{array}$ & $\begin{array}{l}-0.18(-0.59 \text { to } 0.23) \\
-0.78(-1.22 \text { to }-0.33) \\
-0.60(-0.98 \text { to }-0.22)\end{array}$ ** \\
\hline & CLG & $2.68 \pm 0.94$ & $2.86 \pm 1.18$ & $3.08 \pm 1.00$ & $3.65 \pm 1.30$ & $\begin{array}{l}\text { (a) } \\
\text { (b) } \\
\text { (c) }\end{array}$ & $\begin{array}{l}-0.18(-0.52 \text { to } 0.16) \\
-0.40(-0.84 \text { to } 0.04) \\
-0.98(-1.50 \text { to }-0.46)\end{array}$ & $\begin{array}{l}\text { (a) } \\
\text { (b) } \\
\text { (c) }\end{array}$ & $\begin{array}{l}-0.22(-0.64 \text { to } 0.20) \\
-0.80(-1.25 \text { to }-0.34) \text { ** } \\
-0.58(-0.97 \text { to }-0.18)^{* *}\end{array}$ \\
\hline \multirow{2}{*}{ Tibial muscle } & CLG + MNNM & $6.91 \pm 3.06$ & $6.92 \pm 3.16$ & $7.77 \pm 2.96$ & $7.95 \pm 2.78$ & $\begin{array}{l}\text { (a) } \\
\text { (b) } \\
\text { (c) }\end{array}$ & $\begin{array}{l}-0.01(-0.56 \text { to } 0.55) \\
-0.86(-1.75 \text { to } 0.03) \\
-1.04(-1.93 \text { to }-0.15)^{*}\end{array}$ & $\begin{array}{l}\text { (a) } \\
\text { (b) } \\
\text { (c) }\end{array}$ & $\begin{array}{l}-0.85(-1.78 \text { to } 0.08) \\
-1.04(-1.88 \text { to }-0.19) \\
-0.18(-0.98 \text { to } 0.61)\end{array}$ \\
\hline & CLG & $7.24 \pm 3.14$ & $7.88 \pm 3.24$ & $7.59 \pm 3.04$ & $7.80 \pm 2.85$ & $\begin{array}{l}\text { (a) } \\
\text { (b) } \\
\text { (c) }\end{array}$ & $\begin{array}{l}-0.64(-1.20 \text { to }-0.08)^{*} \\
-0.35(-1.26 \text { to } 0.56) \\
-0.55(-1.46 \text { to } 0.36)\end{array}$ & $\begin{array}{l}\text { (a) } \\
\text { (b) } \\
\text { (c) }\end{array}$ & $\begin{array}{l}0.29(-0.66 \text { to } 1.24) \\
0.09(-0.78 \text { to } 0.95) \\
-0.20(-1.02 \text { to } 0.61)\end{array}$ \\
\hline
\end{tabular}

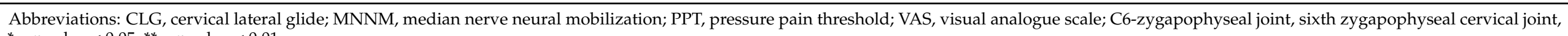
${ }^{*}=p$ value $<0.05, * *=p$ value $<0.01$.

Table 4. Multiple comparisons for active cervical range of motion. Values are the mean \pm standard deviation unless otherwise indicated.

\begin{tabular}{|c|c|c|c|c|c|c|c|c|c|}
\hline \multirow[b]{2}{*}{ CROM (Degrees) } & \multirow[b]{2}{*}{ Group } & \multirow[b]{2}{*}{ Baseline } & \multirow[b]{2}{*}{$\begin{array}{c}\text { Post-Intervention } \\
5 \mathrm{~min}\end{array}$} & \multirow[b]{2}{*}{$\begin{array}{l}\text { Follow-Up } \\
2 \text { Weeks }\end{array}$} & \multirow[b]{2}{*}{$\begin{array}{l}\text { Follow-up } \\
4 \text { Weeks }\end{array}$} & \multicolumn{2}{|c|}{ Mean Difference $(95 \%$ CI) } & \multicolumn{2}{|c|}{ Mean Difference $(95 \% \mathrm{CI})$} \\
\hline & & & & & & $\begin{array}{l}\text { (a) } \\
\text { (b) } \\
\text { (c) }\end{array}$ & $\begin{array}{l}\text { Baseline vs Post } \\
\text { Baseline vs 2-Weeks } \\
\text { Baseline vs 4-Weeks }\end{array}$ & $\begin{array}{l}\text { (a) } \\
\text { (b) } \\
\text { (c) }\end{array}$ & $\begin{array}{l}\text { Post vs 2-Weeks } \\
\text { Post vs } 4 \text {-Weeks } \\
\text { 2-Weeks vs } 4 \text {-Weeks }\end{array}$ \\
\hline \multirow{2}{*}{$\begin{array}{l}\text { Flexion/ } \\
\text { Extension }\end{array}$} & CLG + MNNM & $101.51 \pm 23.53$ & $100.86 \pm 23.76$ & $100.21 \pm 20.35$ & $101.71 \pm 17.74$ & $\begin{array}{l}\text { (a) } \\
\text { (b) } \\
\text { (c) }\end{array}$ & $\begin{array}{l}0.65(-5.19 \text { to } 6.50) \\
1.30(-6.58 \text { to } 9.18) \\
-0.20(-8.33 \text { to } 7.93)\end{array}$ & $\begin{array}{l}\text { (a) } \\
\text { (b) } \\
\text { (c) }\end{array}$ & $\begin{array}{l}0.65(-6.48 \text { to } 7.77) \\
-0.85(-8.04 \text { to } 6.33) \\
-1.50(-7.95 \text { to } 4.95)\end{array}$ \\
\hline & CLG & $105.37 \pm 24.12$ & $109.14 \pm 24.35$ & $108.76 \pm 20.86$ & $107.65 \pm 18.18$ & $\begin{array}{l}\text { (a) } \\
\text { (b) } \\
\text { (c) }\end{array}$ & $\begin{array}{l}-3.77(-9.76 \text { to } 2.22) \\
-3.39(-11.47 \text { to } 4.69) \\
-2.28(-10.61 \text { to } 6.05)\end{array}$ & $\begin{array}{l}\text { (a) } \\
\text { (b) } \\
\text { (c) }\end{array}$ & $\begin{array}{l}0.38(-6.93 \text { to } 7.68) \\
1.49(-5.88 \text { to } 8.85) \\
1.11(-5.51 \text { to } 7.72) \\
\end{array}$ \\
\hline
\end{tabular}


Table 4. Cont.

\begin{tabular}{|c|c|c|c|c|c|c|c|c|c|}
\hline \multirow[b]{2}{*}{ CROM (Degrees) } & \multirow[b]{2}{*}{ Group } & \multirow[b]{2}{*}{ Baseline } & \multirow[b]{2}{*}{$\begin{array}{l}\text { Post-Intervention } \\
\qquad 5 \mathrm{~min}\end{array}$} & \multirow[b]{2}{*}{$\begin{array}{l}\text { Follow-Up } \\
2 \text { Weeks }\end{array}$} & \multirow[b]{2}{*}{$\begin{array}{l}\text { Follow-up } \\
4 \text { Weeks }\end{array}$} & \multicolumn{2}{|c|}{ Mean Difference (95\% CI) } & \multicolumn{2}{|c|}{ Mean Difference (95\% CI) } \\
\hline & & & & & & $\begin{array}{l}\text { (a) } \\
\text { (b) } \\
\text { (c) }\end{array}$ & $\begin{array}{l}\text { Baseline vs Post } \\
\text { Baseline vs } 2 \text {-Weeks } \\
\text { Baseline vs } 4 \text {-Weeks }\end{array}$ & $\begin{array}{l}\text { (a) } \\
\text { (b) } \\
\text { (c) }\end{array}$ & $\begin{array}{l}\text { Post vs 2-Weeks } \\
\text { Post vs 4-Weeks } \\
\text { 2-Weeks vs 4-Weeks }\end{array}$ \\
\hline \multirow{2}{*}{$\begin{array}{l}\text { Homolateral } \\
\text { Rotation }\end{array}$} & CLG + MNNM & $55.26 \pm 13.08$ & $58.23 \pm 13.40$ & $54.82 \pm 11.49$ & $55.94 \pm 12.07$ & $\begin{array}{l}\text { (a) } \\
\text { (b) } \\
\text { (c) }\end{array}$ & $\begin{array}{l}-2.98(-7.43 \text { to } 1.48) \\
0.44(-4.52 \text { to } 5.39) \\
-0.68(-5.16 \text { to } 3.80)\end{array}$ & $\begin{array}{l}\text { (a) } \\
\text { (b) } \\
\text { (c) }\end{array}$ & $\begin{array}{l}0.41(-1.53 \text { to } 8.35) \\
2.30(-2.93 \text { to } 7.52) \\
-1.12(-5.13 \text { to } 2.90)\end{array}$ \\
\hline & CLG & $57.87 \pm 13.41$ & $60.11 \pm 13.74$ & $59.53 \pm 11.78$ & $62.83 \pm 12.38$ * & $\begin{array}{l}\text { (a) } \\
\text { (b) } \\
\text { (c) }\end{array}$ & $\begin{array}{l}-2.24(-6.81 \text { to } 2.33) \\
-1.66(-6.74 \text { to } 3.43) \\
-4.95(-9.55 \text { to }-0.36)\end{array}$ & $\begin{array}{l}\text { (a) } \\
\text { (b) } \\
\text { (c) }\end{array}$ & $\begin{array}{l}0.58(-4.49 \text { to } 5.65) \\
-2.72(-8.07 \text { to } 2.64) \\
-3.30(-7.41 \text { to } 0.82)\end{array}$ \\
\hline \multirow{2}{*}{$\begin{array}{l}\text { Contralateral } \\
\text { Rotation }\end{array}$} & CLG + MNNM & $56.17 \pm 12.05$ & $60.18 \pm 12.71$ & $57.45 \pm 11.70$ & $55.07 \pm 12.38$ & $\begin{array}{l}\text { (a) } \\
\text { (b) } \\
\text { (c) }\end{array}$ & $\begin{array}{l}-4.01(-8.64 \text { to } 0.62) \\
-1.27(-6.08 \text { to } 3.53) \\
1.10(-3.42 \text { to } 5.61)\end{array}$ & $\begin{array}{l}\text { (a) } \\
\text { (b) } \\
\text { (c) }\end{array}$ & $\begin{array}{l}2.74(-2.19 \text { to } 7.66) \\
5.11(0.39 \text { to } 9.82)^{*} \\
2.37(-1.28 \text { to } 6.02)\end{array}$ \\
\hline & CLG & $59.51 \pm 12.35$ & $61.22 \pm 13.03$ & $61.30 \pm 12.00$ & $62.83 \pm 12.70$ * & $\begin{array}{l}\text { (a) } \\
\text { (b) } \\
\text { (c) }\end{array}$ & $\begin{array}{l}-1.71(-6.46 \text { to } 3.04) \\
-1.80(-6.72 \text { to } 3.13) \\
-3.32(-7.95 \text { to } 1.31)\end{array}$ & $\begin{array}{l}\text { (a) } \\
\text { (b) } \\
\text { (c) }\end{array}$ & $\begin{array}{l}-0.09(-5.13 \text { to } 4.96) \\
-1.61(-6.44 \text { to } 3.23) \\
-1.52(-5.27 \text { to } 2.22)\end{array}$ \\
\hline \multirow{2}{*}{$\begin{array}{l}\text { Homlateral } \\
\text { side flexion }\end{array}$} & CLG + MNNM & $33.81 \pm 9.42$ & $35.65 \pm 11.20$ & $34.03 \pm 9.65$ & $35.12 \pm 8.77$ & $\begin{array}{l}\text { (a) } \\
\text { (b) } \\
\text { (c) }\end{array}$ & $\begin{array}{l}-1.84(-5.19 \text { to } 1.51) \\
-0.22(-3.54 \text { to } 3.10) \\
-1.31(-4.71 \text { to } 2.09)\end{array}$ & $\begin{array}{l}\text { (a) } \\
\text { (b) } \\
\text { (c) }\end{array}$ & $\begin{array}{l}1.62(-2.06 \text { to } 5.31) \\
0.53(-3.35 \text { to } 4.41) \\
-1.09(-4.06 \text { to } 1.88)\end{array}$ \\
\hline & CLG & $33.85 \pm 9.66$ & $36.93 \pm 11.48$ & $34.35 \pm 9.89$ & $35.69 \pm 8.99$ & $\begin{array}{l}\text { (a) } \\
\text { (b) } \\
\text { (c) }\end{array}$ & $\begin{array}{l}-3.08(-6.51 \text { to } 0.35) \\
-0.50(-3.90 \text { to } 2.90) \\
-1.84(-5.32 \text { to } 1.65)\end{array}$ & $\begin{array}{l}\text { (a) } \\
\text { (b) } \\
\text { (c) }\end{array}$ & $\begin{array}{l}2.58(-1.19 \text { to } 6.36) \\
1.24(-2.73 \text { to } 5.22) \\
-1.34(-4.38 \text { to } 1.71) \\
\end{array}$ \\
\hline \multirow{2}{*}{$\begin{array}{l}\text { Contralateral } \\
\text { side flexion }\end{array}$} & CLG + MNNM & $33.12 \pm 10.93$ & $34.45 \pm 10.86$ & $34.60 \pm 10.27$ & $36.52 \pm 9.86$ & $\begin{array}{l}\text { (a) } \\
\text { (b) } \\
\text { (c) }\end{array}$ & $\begin{array}{l}-1.33(-4.91 \text { to } 2.25) \\
-1.48(-6.03 \text { to } 3.06) \\
-3.40(-7.56 \text { to } 0.76)\end{array}$ & $\begin{array}{l}\text { (a) } \\
\text { (b) } \\
\text { (c) }\end{array}$ & $\begin{array}{l}-0.15(-3.69 \text { to } 3.39) \\
-2.07(-5.57 \text { to } 1.44) \\
-1.92(-5.67 \text { to } 1.84)\end{array}$ \\
\hline & CLG & $34.61 \pm 11.21$ & $35.36 \pm 11.14$ & $35.93 \pm 10.53$ & $37.00 \pm 10.10$ & $\begin{array}{l}\text { (a) } \\
\text { (b) } \\
\text { (c) }\end{array}$ & $\begin{array}{l}-0.76(-4.43 \text { to } 2.92) \\
-1.32(-5.98 \text { to } 3.34) \\
-2.39(-6.65 \text { to } 1.88)\end{array}$ & $\begin{array}{l}\text { (a) } \\
\text { (b) } \\
\text { (c) }\end{array}$ & $\begin{array}{l}-0.57(-4.19 \text { to } 3.06) \\
-1.63(-5.23 \text { to } 1.96) \\
-1.07(-4.92 \text { to } 2.78)\end{array}$ \\
\hline
\end{tabular}

Abbreviations: CLG, cervical lateral glide; MNNM, median nerve neural mobilization; CROM, cervical range of motion. ${ }^{*}=p$ value $<0.05$. 
Table 5. Multiple comparisons for neck disability and kinesiophobia. Values are the mean \pm standard deviation unless otherwise indicated.

\begin{tabular}{|c|c|c|c|c|c|c|}
\hline & & & & & $\mathrm{Me}$ & n Difference (95\% CI) \\
\hline & Group & Baseline & $\begin{array}{c}\text { Follow-up } \\
2 \text { Weeks }\end{array}$ & $\begin{array}{c}\text { Follow-up } \\
4 \text { Weeks }\end{array}$ & $\begin{array}{l}\text { (a) } \\
\text { (b) } \\
\text { (c) }\end{array}$ & $\begin{array}{l}\text { Baseline vs } 2 \text {-weeks } \\
\text { Baseline vs } 4 \text {-weeks } \\
\text { 2-weeks vs } 4 \text {-weeks }\end{array}$ \\
\hline \multirow{2}{*}{ NDI } & CLG + MNNM & $17.66 \pm 7.94$ & $15.69 \pm 7.31$ & $13.88 \pm 6.98$ & $\begin{array}{l}\text { (a) } \\
\text { (b) } \\
\text { (c) }\end{array}$ & $\begin{array}{l}1.97(0.28 \text { to } 3.66)^{*} \\
3.79(1.63 \text { to } 5.95) * * \\
1.82(0.38 \text { to } 3.26)^{* *}\end{array}$ \\
\hline & CLG & $16.54 \pm 8.14$ & $14.16 \pm 7.49$ & $11.73 \pm 7.16$ & $\begin{array}{l}\text { (a) } \\
\text { (b) } \\
\text { (c) }\end{array}$ & $\begin{array}{l}2.38(0.65 \text { to } 4.11)^{* *} \\
4.81(2.60 \text { to } 7.03)^{* *} \\
2.44(0.96 \text { to } 3.91)^{* *}\end{array}$ \\
\hline \multirow{2}{*}{ TSK-11 } & CLG + MNNM & $27.93 \pm 7.78$ & $25.21 \pm 7.78$ & $25.43 \pm 7.70$ & $\begin{array}{l}\text { (a) } \\
\text { (b) } \\
\text { (c) }\end{array}$ & $\begin{array}{l}2.73(0.80 \text { to } 4.65) * * \\
2.50(0.33 \text { to } 4.67) * \\
-0.23(-2.14 \text { to } 1.69)\end{array}$ \\
\hline & CLG & $28.35 \pm 7.98$ & $26.81 \pm 7.98$ & $24.60 \pm 7.90$ & $\begin{array}{l}\text { (a) } \\
\text { (b) } \\
\text { (c) }\end{array}$ & $\begin{array}{l}1.54(-0.44 \text { to } 3.51) \\
3.75(1.52 \text { to } 5.97) * * \\
2.21(0.25 \text { to } 4.18)^{*}\end{array}$ \\
\hline
\end{tabular}

Abbreviations: CLG, cervical lateral glide; NDI, neck disability index; MNNM, median nerve neural mobilization; TSK-11, Tampa Scale of Kinesiophobia. ${ }^{*}=p$ value $<0.05,{ }^{* *}=p$ value $<0.01$.

\section{Discussion}

Neural mobilization approaches have been demonstrated to be effective for improving cervicobrachial pain conditions [17-20]. However, to our knowledge, this is the first study to assess the effectiveness of the combination of two neural mobilization techniques for improving neural mechanosensitivity, pain, and function in patients with NP. Here we demonstrate a positive effect for improving median nerve mechanosensitivity, regardless of whether the application of lateral cervical glide is applied in combination with the mobilization of the median nerve. There are no differences between the two forms. These findings suggest that the application of only one neural mobilization technique might be sufficient to improve NP in clinical practice. Hence, the time spent on manual therapy techniques could be reduced in favor of other alternative approaches, such as therapeutic exercise or therapeutic education [60].

\subsection{Median Nerve Mechanosensitivity}

The amount of improvement obtained in this study is similar or slightly higher than those obtained in other studies. Vicenzino et al. [61] found that patients suffering from epicondylalgia, subjected to a single session of lateral cervical sliding ( $1.5 \mathrm{~min}$ ) obtained an improvement of $26.03 \%\left(0.45 \mathrm{~kg} / \mathrm{cm}^{2}\right)$. In our study, we detected an improvement of greater magnitude in the lateral cervical glide group with an increase of $34.16 \%\left(0.96 \mathrm{~kg} / \mathrm{cm}^{2}\right)$ at 30 days of follow-up, while in the group in which lateral cervical glide was combined with the mobilization of the median nerve the percentage of improvement was just $15.94 \%$ $\left(0.44 \mathrm{~kg} / \mathrm{cm}^{2}\right)$. Another more recent study [62] applied three sessions of a combined protocol of manual soft-tissue therapy together with neural mobilization exercises to patients with Carpal tunnel syndrome. These authors detected an improvement in the PPT of the median nerve region of $23.6 \%\left(0.80 \mathrm{~kg} / \mathrm{cm}^{2}\right)$ that remains 1 year after follow-up. Bialosky et al. [63], on the other hand, did not achieve an improvement in mechanical hypoalgesia when applying exercises to mobilize the median nerve (during nine sessions) against a placebo group in patients with carpal tunnel syndrome, although the group subjected to neural mobilization obtained a significant improvement $(41.26 \%)$, and superior to that found in our study (34.16\%). These differences might be because the other study had a greater number of sessions. Finally, a study that applied a session of neural mobilization 
of the median nerve in patients with NP [64] found contradictory results; there were no improvements in mechanical hypoalgesia in the cervical region. Based on these findings, we propose that the effects of neural mobilization are effects that are best in the body area where it is applied. It could be that the differences with that study are that they did one session while we did two. Moreover, in this research, we found improvements in the mechanical hypoalgesia of the median nerve on the contralateral side, producing improvements of $32.2 \%$ and $29.65 \%$ in the group of CLG + MNNM and CLG only, respectively.

In relation to neural mechanosensitivity evaluated by the neural tension test neural measured by the range of motion of the elbow extension, Coppieters et al. [65] found an improvement of $13.86 \%$ (19 degrees) in patients with cervicobrachial neuralgia when a single session of lateral cervical glide was applied, these results are consistent with those reported in our study. These results are in line with our findings, with a slightly greater improvement in the CLG + MNNM group than CLG group $(26.62 \%$ and $19.11 \%$, respectively). In contrast, another study [66] found an improvement of only $6 \%$ after the application of nine sessions of neural mobilization on the median nerve in healthy patients. It is possible that this difference is because they were not patients suffering from pain.

\subsection{Widespread Pain Assessment}

In relation to the NP intensity, although no differences were found between the groups, these presented an improvement of $27.67 \%$ (1.2 points) in the CLG + MNNM group and $31.49 \%$ (1.3 points) in the CLG group. These results are lower than those obtained by Rodríguez et al. [19] in which they applied neural mobilization of the median nerve to patients with cervicobrachial neuralgia and obtained a $52.76 \%$ improvement (3.08 points], which is much more clinically relevant than the improvements obtained in our study. Although the changes obtained reached the minimally detectable threshold (1.15 points) [67]. The number of sessions that applied was much higher ( 20 sessions) compared to the two sessions that we applied in our study, which might account for the different findings. Another study [20] in which the authors applied 30 sessions of the lateral cervical sliding technique also found effects on pain intensity superior to our study, with an improvement that reached $35.5 \%$ (2.16 points). Finally, in another study [22] that compared 30 sessions of neural mobilization of the median nerve against lateral cervical glide in patients with cervicobrachial neuralgia, the authors found an improvement superior to that of our study, reaching $36.06 \%$ (2.2 points) in the group subjected to lateral cervical glide and $46.15 \%$ ( 3 points) in the group of neural mobilization of the median nerve. Again, we believe that the number of sessions is the main cause of this difference. In addition, our study used neural gliding in accordance with prior experimental research which showed that neural mobilization generated immediate widespread hypoalgesic effects in more body sites compared to neural stretching [68].

\subsection{Cervical Function and Kinesiofobia}

Although there was an improvement in function, this was inferior to that reported in other studies. The improvement in rotation in the CLG group only reached improvements of $8.57 \%$ (4.96 degrees) and $5.57 \%$ (3.32 degrees) in the contralateral rotation. These data are contradictory to those found in several previous studies $[19,20,22]$, achieving a $12.1 \%$ improvement in the cervical range of motion in patients with cervicobrachial neuralgia after application of median nerve neural mobilization [19], and an 18\% (10 degrees) improvement when lateral cervical slippage was applied [20,22]. This difference could be explained by the fact that, in those three previous studies, 30 sessions were applied as opposed to the two sessions we have applied in our study. Nevertheless, the follow-up presented the same duration (30 days). On the other hand, neck disability was improved in both groups, but neither group was superior to the other according to the results obtained in the NDI. This outcome was assessed with NDI. NDI is the preferred tool for the evaluation of disability levels [49,69] in patients suffering from non-specific NP, with [19-22] or without irradiation symptoms $[50,51,70]$. 
Few studies have previously included the kinesiophobia variable. The two studies we could identify in which neural mobilization is applied used kinesiophobia as a confusion variable to test how it interfered with the improvement of pain in healthy subjects [66] or pain modulation in patients with NP [64]. In our study, all patients improved in kinesiophobia although with discrete changes (2.5 points $(8.95 \%)$ in the CLG + MNNM group and 3.75 points (13.22\%) in the CLG group).

\subsection{Limitations}

This study has some limitations. First, the lack of a control group and/or placebo group means that we cannot determine the effects of these non-specific interventions or test whether they are superior to the absence of treatment. Second, only kinesiophobia was measured, so we cannot determine the effects on other psychological factors. The third limitation is that only two treatment sessions were carried out. If more sessions had been applied, we could have found other effects. Another limitation is the short-term (30 days) follow-up of patients; therefore, we cannot know the effects in the medium and long term.

\section{Conclusions}

Adding neural mobilization of the median nerve to the cervical lateral sliding technique is not superior to performing it alone in improving the mechanosensitivity of the median nerve, expanding pain, NP intensity, improving function, and kinesiophobia. Only two treatment sessions and the short follow-up are important issues, therefore justifying further studies to answer the research question with better methodology.

Author Contributions: Conceptualization, D.M.-V., D.P.-M. and P.M.-L.; methodology D.M.-V., D.P.M. and A.A.-R. software, D.M.-V., D.R.-S., C.C.-L., P.M.-L., A.A.-R. and I.L.-d.-U.-V.; validation, all authors.; formal analysis, D.M.-V., D.P.-M. and J.F-C.; investigation, all authors.; resources, D.P.-M.; data curation, D.M.-V., D.P.-M. and J.F.-C.; writing-original draft preparation, D.M.-V., D.P.-M., D.R.-S., C.C.-L., I.L.-d.-U.-V. and J.F.-C.; writing-review and editing, all authors.; visualization, J.F.-C.; supervision, all authors.; project administration, D.M.-V. and D.P.-M.; funding acquisition, D.M.-V. and D.P.-M. All authors have read and agreed to the published version of the manuscript.

Funding: This project has not received research funding.

Institutional Review Board Statement: The study protocol was approved by Ethics Committee for Clinical Research of the Principe de Asturias Hospital (Code: OE 19/2013).

Informed Consent Statement: Informed consent was obtained from all subjects involved in the study. Written informed consent for publication must be obtained from participating patients who can be identified (including by the patients themselves). Written informed consent has been obtained from the patient(s) to publish this paper.

Data Availability Statement: Data available on request due to privacy and ethical restrictions.

Conflicts of Interest: The authors certify that they have no affiliations with or financial involvement in any organization or entity with a direct financial interest in the subject matter or materials discussed in the article.

\section{References}

1. Palacios-Ceña, D.; Alonso-Blanco, C.; Hernández-Barrera, V.; Carrasco-Garrido, P.; Jiménez-García, R.; Fernández-de-Las-Peñas, C. Prevalence of neck and low back pain in community-dwelling adults in Spain: An updated population-based national study (2009/10-2011/12). Eur. Spine J. 2015, 24, 482-492. [CrossRef]

2. GBD 2013 DALYs; HALE Collaborators; Murray, C.J.L.; Barber, R.M.; Foreman, K.J.; Abbasoglu Ozgoren, A.; Abd-Allah, F.; Abera, S.F.; Aboyans, V.; Abraham, J.P.; et al. Global, regional, and national disability-adjusted life years (DALYs) for 306 diseases and injuries and healthy life expectancy (HALE) for 188 countries, 1990-2013: Quantifying the epidemiological transition. Lancet Lond. Engl. 2015, 386, 2145-2191. [CrossRef]

3. Hoy, D.; March, L.; Woolf, A.; Blyth, F.; Brooks, P.; Smith, E.; Vos, T.; Barendregt, J.; Blore, J.; Murray, C.; et al. The global burden of neck pain: Estimates from the global burden of disease 2010 study. Ann. Rheum. Dis. 2014, 73, 1309-1315. [CrossRef] 
4. Carroll, L.J.; Hogg-Johnson, S.; van der Velde, G.; Haldeman, S.; Holm, L.W.; Carragee, E.J.; Hurwitz, E.L.; Côté, P.; Nordin, M.; Peloso, P.M.; et al. Course and prognostic factors for neck pain in the general population: Results of the Bone and Joint Decade 2000-2010 Task Force on Neck Pain and Its Associated Disorders. J. Manip. Physiol. Ther. 2009, 32, S87-S96. [CrossRef] [PubMed]

5. Hogg-Johnson, S.; van der Velde, G.; Carroll, L.J.; Holm, L.W.; Cassidy, J.D.; Guzman, J.; Côté, P.; Haldeman, S.; Ammendolia, C.; Carragee, E.; et al. The burden and determinants of neck pain in the general population: Results of the Bone and Joint Decade 2000-2010 Task Force on Neck Pain and Its Associated Disorders. J. Manip. Physiol. Ther. 2009, 32, S46-S60. [CrossRef]

6. Haldeman, S.; Carroll, L.; Cassidy, J.D. Findings from the bone and joint decade 2000 to 2010 task force on neck pain and its associated disorders. J. Occup. Environ. Med. 2010, 52, 424-427. [CrossRef]

7. Van Hecke, O.; Austin, S.K.; Khan, R.A.; Smith, B.H.; Torrance, N. Neuropathic pain in the general population: A systematic review of epidemiological studies. PAIN 2014, 155, 654-662. [CrossRef]

8. Gangavelli, R.; Nair, N.S.; Bhat, A.K.; Solomon, J.M. Cervicobrachial pain-How Often is it Neurogenic? J. Clin. Diagn. Res. 2016, 10, YC14-YC16. [CrossRef]

9. Kozma, C.M.; Provenzano, D.A.; Slaton, T.L.; Patel, A.A.; Benson, C.J. Complexity of Pain Management Among Patients with Nociceptive or Neuropathic Neck, Back, or Osteoarthritis Diagnoses. J. Manag. Care Pharm. 2014, 20, 455-466. [CrossRef] [PubMed]

10. Gross, A.R.; Aker, P.D.; Goldsmith, C.H.; Peloso, P. Physical medicine modalities for mechanical neck disorders. Cochrane Database Syst. Rev. 2000, 18, CD000961. [CrossRef] [PubMed]

11. Aker, P.D.; Gross, A.R.; Goldsmith, C.H.; Peloso, P. Conservative management of mechanical neck pain: Systematic overview and meta-analysis. BMJ 1996, 313, 1291-1296. [CrossRef]

12. Thoomes, E.J.; Scholten-Peeters, W.; Koes, B.; Falla, D.; Verhagen, A.P. The effectiveness of conservative treatment for patients with cervical radiculopathy: A systematic review. Clin. J. Pain 2013, 29, 1073-1086. [CrossRef]

13. Thoomes, E.J. Effectiveness of manual therapy for cervical radiculopathy, a review. Chiropr. Man. Therap. 2016, 24, 45. [CrossRef]

14. Boyles, R.; Toy, P.; Mellon, J.; Hayes, M.; Hammer, B. Effectiveness of manual physical therapy in the treatment of cervical radiculopathy: A systematic review. J. Man. Manip. Ther. 2011, 19, 135-142. [CrossRef]

15. Ellis, R.F.; Hing, W.A. Neural mobilization: A systematic review of randomized controlled trials with an analysis of therapeutic efficacy. J. Man. Manip. Ther. 2008, 16, 8-22. [CrossRef]

16. Basson, A.; Olivier, B.; Ellis, R.; Coppieters, M.; Stewart, A.; Mudzi, W. The Effectiveness of Neural Mobilization for Neuromusculoskeletal Conditions: A Systematic Review and Meta-analysis. J. Orthop. Sport Phys. Ther. 2017, 47, 593-615. [CrossRef]

17. Costello, M.; Puentedura, E.L.J.; Cleland, J.; Ciccone, C.D. The immediate effects of soft tissue mobilization versus therapeutic ultrasound for patients with neck and arm pain with evidence of neural mechanosensitivity: A randomized clinical trial. J. Man. Manip. Ther. 2016, 24, 128-140. [CrossRef]

18. Kim, D.-G.; Chung, S.H.; Jung, H.B. The effects of neural mobilization on cervical radiculopathy patients' pain, disability, ROM, and deep flexor endurance. J. Back Musculoskelet. Rehabil. 2017, 30, 951-959. [CrossRef]

19. Rodríguez-Sanz, D.; López-López, D.; Unda-Solano, F.; Romero-Morales, C.; Sanz-Corbalán, I.; Beltran-Alacreu, H.; Calvo-Lobo, C. Effects of Median Nerve Neural Mobilization in Treating Cervicobrachial Pain: A Randomized Waiting List-Controlled Clinical Trial. Pain Pract. 2018, 18, 431-442. [CrossRef]

20. Rodríguez-Sanz, D.; Calvo-Lobo, C.; Unda-Solano, F.; Sanz-Corbalán, I.; Romero-Morales, C.; López-López, D. Cervical Lateral Glide Neural Mobilization Is Effective in Treating Cervicobrachial Pain: A Randomized Waiting List Controlled Clinical Trial. Pain Med. 2017, 18, 2492-2503. [CrossRef]

21. Sanz, D.R.; Solano, F.U.; López, D.L.; Corbalan, I.S.; Morales, C.R.; Lobo, C.C. Effectiveness of median nerve neural mobilization versus oral ibuprofen treatment in subjects who suffer from cervicobrachial pain: A randomized clinical trial. Arch. Med. Sci. 2017, 14, 871-879. [CrossRef]

22. Calvo-Lobo, C.; Unda-Solano, F.; López-López, D.; Sanz-Corbalán, I.; Romero-Morales, C.; Palomo-López, P.; Seco-Calvo, J.; Rodríguez-Sanz, D. Is pharmacologic treatment better than neural mobilization for cervicobrachial pain? A randomized clinical trial. Int. J. Med. Sci. 2018, 15, 456-465. [CrossRef]

23. Isabel de-la-Llave-Rincón, A.; Puentedura, E.J.; Fernández-de-Las-Peñas, C. Clinical presentation and manual therapy for upper quadrant musculoskeletal conditions. J. Man. Manip. Ther. 2011, 19, 201-211. [CrossRef]

24. Cobos-Carbó, A.; Augustovski, F. Declaración CONSORT 2010, actualización de la lista de comprobación para informar ensayos clínicos aleatorizados de grupos paralelos. Med. Clin. Barc. 2011, 137, 213-215. [CrossRef]

25. World-Medical-Association. World Medical Association declaration of Helsinki: Ethical principles for medical research involving human subjects. JAMA J. Am. Med. Assoc. 2013, 310, 2191-2194. [CrossRef]

26. Schmid, A.B.; Brunner, F.; Luomajoki, H.; Held, U.; Bachmann, L.M.; Künzer, S.; Coppieters, M.W. Reliability of clinical tests to evaluate nerve function and mechanosensitivity of the upper limb peripheral nervous system. BMC Musculoskelet. Disord. 2009, 10, 11. [CrossRef]

27. Wainner, R.S.; Fritz, J.M.; Irrgang, J.J.; Boninger, M.L.; Delitto, A.; Allison, S. Reliability and diagnostic accuracy of the clinical examination and patient self-report measures for cervical radiculopathy. Spine Phila Pa 1976 2003, 28, 52-62. [CrossRef]

28. Kleinrensink, G.J.; Stoeckart, R.; Vleeming, A.; Snijders, C.J.; Mulder, P.G.H. Mechanical tension in the median nerve. The effects of joint positions. Clin. Biomech. Bristol. Avon. 1995, 10, 240-244. [CrossRef] 
29. Nee, R.J.; Jull, G.A.; Vicenzino, B.; Coppieters, M.W. The Validity of Upper-Limb Neurodynamic Tests for Detecting Peripheral Neuropathic Pain. J. Orthop. Sport Phys. Ther. 2012, 42, 413-424. [CrossRef]

30. Coppieters, M.; Stappaerts, K.; Janssens, K.; Jull, G. Reliability of detecting "onset of pain" and "submaximal pain" during neural provocation testing of the upper quadrant. Physiother. Res. Int. 2002, 7, 146-156. [CrossRef]

31. Hjermstad, M.J.; Fayers, P.M.; Haugen, D.F.; Caraceni, A.; Hanks, G.W.; Loge, J.H.; Fainsinger, R.; Aass, N.; Kaasa, S. Studies Comparing Numerical Rating Scales, Verbal Rating Scales, and Visual Analogue Scales for Assessment of Pain Intensity in Adults: A Systematic Literature Review. J. Pain Symptom. Manag. 2011, 41, 1073. [CrossRef]

32. Kahl, C.; Cleland, J.A. Visual analogue scale, numeric pain rating scale and the McGill pain Questionnaire: An overview of psychometric properties. Phys. Ther. Rev. 2005, 10, 123-128. [CrossRef]

33. Rosier, E.M.; Iadarola, M.J.; Coghill, R.C. Reproducibility of pain measurement and pain perception. Pain 2002, 98, 205-216. [CrossRef]

34. Barbero, M.; Moresi, F.; Leoni, D.; Gatti, R.; Egloff, M.; Falla, D. Test-retest reliability of pain extent and pain location using a novel method for pain drawing analysis. Eur. J. Pain. 2015, 19, 1129-1138. [CrossRef]

35. Breivik, H.; Borchgrevink, P.C.; Allen, S.M.; Rosseland, L.A.; Romundstad, L.; Hals, E.K.B.; Kvarstein, G.; Stubhaug, A. Assessment of pain. Br. J. Anaesth. 2008, 101, 17-24. [CrossRef]

36. Rebbeck, T.; Moloney, N.; Azoory, R.; Hubscher, M.; Waller, R.; Gibbons, R.; Beales, D. Clinical Ratings of Pain Sensitivity Correlate With Quantitative Measures in People With Chronic Neck Pain and Healthy Controls: Cross-Sectional Study. Phys. Ther. 2015, 95, 1536-1546. [CrossRef]

37. Backonja, M.-M.; Walk, D.; Edwards, R.R.; Sehgal, N.; Moeller-Bertram, T.; Wasan, A.; Irving, G.; Argoff, C.; Wallace, M. Quantitative sensory testing in measurement of neuropathic pain phenomena and other sensory abnormalities. Clin. J. Pain 2009, 25, 641-647. [CrossRef]

38. Konopka, K.H.; Harbers, M.; Houghton, A.; Kortekaas, R.; van Vliet, A.; Timmerman, W.; den Boer, J.A.; Struys, M.M.R.F.; van Wijhe, M. Bilateral sensory abnormalities in patients with unilateral neuropathic pain; A quantitative sensory testing (QST) study. PLOS ONE 2012, 7, e37524.

39. Loeser, J.; Arendt-Nielsen, L.; Baron, R.; Basbaum, A.; Bond, M.; Breivik, H.; Clauw, D.; De Laat, A.; Dworkin, R.; Giamberardino, M.; et al. Pain Terms, A Current List with Definitions and Notes on Usage. In Classification of Chronic Pain; IASP Press: Seattle, WA, USA, 2011; pp. 209-214.

40. Sterling, M.; Treleaven, J.; Jull, G.A. Responses to a clinical test of mechanical provocation of nerve tissue in whiplash associated disorder. Man. Ther. 2002, 7, 89-94. [CrossRef]

41. Lascurain-Aguirrebena, I.; Newham, D.; Critchley, D.J. Mechanism of action of spinal mobilizations a systematic review. Spine Phila Pa 1976 2016, 41, 159-172. [CrossRef]

42. Salom-Moreno, J.; Ortega-Santiago, R.; Cleland, J.A.; Palacios-Ceña, M.; Truyols-Domínguez, S.; Fernández-de-las-Peñas, C. Immediate changes in neck pain intensity and widespread pressure pain sensitivity in patients with bilateral chronic mechanical neck pain: A randomized controlled trial of thoracic thrust manipulation vs non-thrust mobilization. J. Manip. Physiol. Ther. 2014, 37, 312-319. [CrossRef] [PubMed]

43. Bisset, L.M.; Evans, K.; Tuttle, N. Reliability of 2 protocols for assessing pressure pain threshold in healthy young adults. J. Manip. Physiol. Ther. 2015, 38, 282-287. [CrossRef] [PubMed]

44. Prushansky, T.; Handelzalts, S.; Pevzner, E. Reproducibility of pressure pain threshold and visual analog scale findings in chronic whiplash patients. Clin. J. Pain 2007, 23, 339-345. [CrossRef]

45. Walton, D.M.; Macdermid, J.C.; Nielson, W.; Teasell, R.W.; Chiasson, M.; Brown, L. Reliability, standard error, and minimum detectable change of clinical pressure pain threshold testing in people with and without acute neck pain. J. Orthop. Sports Phys. Ther. 2011, 41, 644-650. [CrossRef]

46. Fischer, A.A. Algometry in Diagnosis of Musculoskeletal Pain and Evaluation of Treatment Outcome: An Update. J. Musculoskelet. Pain 1998, 6, 5-32. [CrossRef]

47. Vanderweeën, L.; Oostendorp, R.A.B.; Vaes, P.; Duquet, W. Pressure algometry in manual therapy. Man. Ther. 1996, 1, 258-265. [CrossRef] [PubMed]

48. Fletcher, J.P.; Bandy, W.D. Intrarater reliability of CROM measurement of cervical spine active range of motion in persons with and without neck pain. J. Orthop. Sports Phys. Ther. 2008, 38, 640-645. [CrossRef]

49. Vernon, H. The Neck Disability Index: State-of-the-art, 1991-2008. J. Manip. Physiol. Ther. 2008, 31, 491-502. [CrossRef]

50. Macdermid, J.C.; Walton, D.M.; Côté, P.; Santaguida, P.L.; Gross, A.; Carlesso, L.; ICON. Use of outcome measures in managing neck pain: An international multidisciplinary survey. Open Orthop. J. 2013, 7, 506. [CrossRef]

51. Murphy, D.R.; Lopez, M. Neck and back pain specific outcome assessment questionnaires in the Spanish language: A systematic literature review. Spine J. 2013, 13, 1667-1674. [CrossRef]

52. Andrade Ortega, J.A.; Delgado Martínez, A.D.; Almécija Ruiz, R. Validation of the Spanish version of the Neck Disability Index. Spine Phila Pa 1976. 2010, 35, E114-E118. [CrossRef] [PubMed]

53. Hudes, K. The Tampa Scale of Kinesiophobia and neck pain, disability and range of motion: A narrative review of the literature. J. Can. Chiropr. Assoc. 2011, 55, 222-232.

54. Gómez-Pérez, L.; López-Martínez, A.E.; Ruiz-Párraga, G.T. Psychometric Properties of the Spanish Version of the Tampa Scale for Kinesiophobia (TSK). J. Pain. 2011, 12, 425-435. [CrossRef] 
55. Roelofs, J.; Goubert, L.; Peters, M.L.; Vlaeyen, J.W.S.; Crombez, G. The Tampa Scale for Kinesiophobia: Further examination of psychometric properties in patients with chronic low back pain and fibromyalgia. Eur. J. Pain 2004, 8, 495-502. [CrossRef]

56. Nee, R.J.; Vicenzino, B.; Jull, G.A.; Cleland, J.A.; Coppieters, M.W. A novel protocol to develop a prediction model that identifies patients with nerve-related neck and arm pain who benefit from the early introduction of neural tissue management. Contemp. Clin. Trials 2011, 32, 760-770. [CrossRef]

57. Guzman, J.; Hurwitz, E.L.; Carroll, L.J.; Haldeman, S.; Cote, P.; Carragee, E.J.; Peloso, P.M.; van der Velde, G.; Holm, L.W.; Hogg-Johnson, S.; et al. A New Conceptual Model of Neck Pain Linking Onset, Course, and Care: The Bone and Joint Decade 2000 -2010 Task Force on Neck Pain and Its Associated Disorders. Spine Phila Pa 1976 2008, 33, S14-S23. [CrossRef]

58. Nee, R.J.; Vicenzino, B.; Jull, G.A.; Cleland, J.A.; Coppieters, M.W. Neural tissue management provides immediate clinically relevant benefits without harmful effects for patients with nerve-related neck and arm pain: A randomised trial. J. Physiother. 2012, 58, 23-31. [CrossRef]

59. Khalil, H.; Quinn, L.; van Deursen, R.; Martin, R.; Rosser, A.; Busse, M. Adherence to Use of a Home-Based Exercise DVD in People With Huntington Disease: Participants' Perspectives. Phys. Ther. 2012, 92, 69-82. [CrossRef]

60. Bernal-Utrera, C.; Gonzalez-Gerez, J.J.; Anarte-Lazo, E.; Rodriguez-Blanco, C. Manual therapy versus therapeutic exercise in non-specific chronic neck pain: A randomized controlled trial. Trials 2020, 21. [CrossRef]

61. Vicenzino, B.; Collins, D.; Wright, A. The initial effects of a cervical spine manipulative physiotherapy treatment on the pain and dysfunction of lateral epicondylalgia. Pain 1996, 68, 69-74. [CrossRef]

62. Fernández-de-las-Peñas, C.; Cleland, J.; Palacios-Ceña, M.; Fuensalida-Novo, S.; Alonso-Blanco, C.; Pareja, J.A.; AlburquerqueSendín, F. Effectiveness of manual therapy versus surgery in pain processing due to carpal tunnel syndrome: A randomized clinical trial. Eur. J. Pain USA 2017, 21, 1266-1276. [CrossRef]

63. Bialosky, J.E.; Bishop, M.D.; Price, D.D.; Robinson, M.E.; Vincent, K.R.; George, S.Z. A randomized sham-controlled trial of a neurodynamic technique in the treatment of carpal tunnel syndrome. J. Orthop. Sports Phys. Ther. 2009, 39, 709-723. [CrossRef]

64. Fernández-Carnero, J.; Sierra-Silvestre, E.; Beltran-Alacreu, H.; Gil-Martínez, A.; La Touche, R. Neural Tension Technique Improves Immediate Conditioned Pain Modulation in Patients with Chronic Neck Pain: A Randomized Clinical Trial. Pain Med. 2019, 20, 1227-1235. [CrossRef]

65. Coppieters, M.W.; Stappaerts, K.H.; Wouters, L.L.; Janssens, K. The immediate effects of a cervical lateral glide treatment technique in patients with neurogenic cervicobrachial pain. J. Orthop. Sports Phys. Ther. 2003, 33, 369-378. [CrossRef] [PubMed]

66. Beneciuk, J.M.; Bishop, M.D.; George, S.Z. Effects of upper extremity neural mobilization on thermal pain sensitivity: A sham-controlled study in asymptomatic participants. J. Orthop. Sports Phys. Ther. 2009, 39, 428-438. [CrossRef]

67. Emshoff, R.; Bertram, S.; Emshoff, I. Clinically important difference thresholds of the visual analog scale: A conceptual model for identifying meaningful intraindividual changes for pain intensity. Pain 2011, 152, 2277-2282. [CrossRef] [PubMed]

68. Beltran-Alacreu, H.; Jiménez-Sanz, L.; Fernández Carnero, J.; La Touche, R. Comparison of Hypoalgesic Effects of Neural Stretching vs Neural Gliding: A Randomized Controlled Trial. J. Manip. Physiol. Ther. 2015, 38, 644-652. [CrossRef]

69. Vernon, H.; Mior, S. The Neck Disability Index: A study of reliability and validity. J. Manip. Physiol. Ther. 1991, 14, 409-415.

70. MacDermid, J.C.; Walton, D.M.; Avery, S.; Blanchard, A.; Etruw, E.; McAlpine, C.; Goldsmith, C.H. Measurement properties of the neck disability index: A systematic review. J. Orthop. Sports Phys. Ther. 2009, 39, 400-417. [CrossRef] [PubMed] 